\title{
Seven years on from the Canadian Airway Focus Group Difficult Airway Guidelines: an observational survey
}

\section{Lignes directrices pour la prise en charge des voies aériennes difficiles du Canadian Airway Focus Group : un sondage observationnel sept ans plus tard}

\author{
Malcom Howard, MD, MSc • Ruediger Noppens, MD, PhD • Nelson Gonzalez, MD, MSc • \\ Philip M. Jones, MD, MSc • Sonja M. Payne, MD, MSc $\mathbb{D}$
}

Received: 14 October 2020/Revised: 24 February 2021/ Accepted: 25 February 2021/Published online: 6 July 2021

(C) Canadian Anesthesiologists' Society 2021

\begin{abstract}
Purpose Numerous clinical guidelines are available for management of the unanticipated difficult airway. It is unclear if practice recommendations are endorsed on regional, local, or individual levels. The objective of this observational study was to examine local and regional use of airway guidelines by anesthesiologists within a hospital partnership in Southwestern Ontario.

Methods Using a paper survey, distributed locally to consultant and trainee anesthesiologists in a tertiary hospital, we examined individual clinical and educational practices regarding guideline use in airway management. Respondents were asked to report which published guideline they used for unanticipated airway difficulty. The effectiveness of dissemination of the national Canadian airway guidelines-the Canadian Airway Focus Group (CAFG) Difficult Airway Guidelines-was examined. We invited anesthesia department heads within
\end{abstract}

This article is accompanied by two editorials. Please see Can J Anesth 2021; this issue.

Supplementary Information The online version contains supplementary material available at https://doi.org/10.1007/s12630021-02056-5.

M. Howard, MD, MSc - R. Noppens, MD, PhD .

N. Gonzalez, MD, MSc · P. M. Jones, MD, MSc .

S. M. Payne, MD, MSc ( $\square)$

Department of Anesthesia \& Perioperative Medicine, University

of Western Ontario, London, ON, Canada

e-mail: sonja.payne@lhsc.on.ca

Department of Epidemiology \& Biostatistics, University of

Western Ontario, London, ON, Canada the regional hospital partnership to complete an electronic survey investigating departmental adoption of guidelines. Results The response rate was 70\% locally (79/112 anesthesiologists) and $52 \%$ regionally (11/21 department heads). Approximately $80 \%$ (64/79) of respondents reported using a formal clinical guideline if unanticipated difficulty with airway management was encountered. Seventy-two per cent of respondents (57/79) were aware of the published CAFG guidelines. Approximately $\quad 30 \% \quad$ (16/51) of consultant anesthesiologists reported using the CAFG guidelines in clinical practice. Within the hospital partnership, 36\% (4/ 11) of departments formally endorsed use of a specific airway management guideline.

Conclusions Despite widespread awareness of the national CAFG Difficult Airway Guidelines, they are not widely adopted in clinical practice. Further research is warranted to explore barriers to adoption of airway management guidelines for both individual anesthesiologists and anesthesia departments.

\section{Résumé}

Objectif De nombreuses lignes directrices cliniques sont disponibles pour la prise en charge des voies aériennes difficiles non anticipées. Nous ne savons pas si ces recommandations de pratique sont suivies aux niveaux régional, local ou individuel. L'objectif de cette étude observationnelle était d'examiner l'utilisation locale et régionale des lignes directrices sur les voies aériennes par des anesthésiologistes provenant d'un partenariat hospitalier dans le sud-ouest de l'Ontario.

Méthode A' l'aide d'une enquête papier, distribuée localement aux anesthésiologistes et aux résidents en 
anesthésiologie d'un hôpital de soins tertiaires, nous avons examiné les pratiques cliniques et éducatives individuelles concernant l'utilisation des lignes directrices dans la prise en charge des voies aériennes. On a demandé aux répondants de divulguer les lignes directrices publiées qu'ils utilisaient lorsqu'ils étaient confrontés à des difficultés imprévues au niveau des voies aériennes. L'efficacité de diffusion des lignes directrices nationales canadiennes sur les voies aériennes-les Lignes directrices sur les voies aériennes difficiles $d u$ Canadian Airway Focus Group (CAFG)-a été examinée. Nous avons invité les chefs des départements d'anesthésiologie au sein du partenariat hospitalier régional à remplir un sondage électronique sur l'adoption des lignes directrices par leur département.

Résultats Le taux de réponse était de $70 \%$ au niveau local (79/112) et de $52 \%$ au niveau régional (11/21 chefs de département). Environ $80 \%$ (64/79) des répondants ont déclaré avoir utilisé des lignes directrices cliniques officielles en cas de difficultés imprévues dans la prise en charge des voies aériennes. Soixante-douze pour cent des répondants (57/79) avaient connaissance des lignes directrices publiées par le CAFG. Environ 30 \% (16/51) des anesthésiologistes ont déclaré avoir utilisé les lignes directrices du CAFG dans leur pratique clinique. Au sein du partenariat hospitalier, $36 \%$ (4/11) des départements ont officiellement appuyé l'utilisation d'une ligne directrice spécifique pour la prise en charge des voies aériennes.

Conclusion Malgré une importante sensibilisation aux Lignes directrices nationales sur les voies aériennes difficiles du CAFG, ces dernières ne sont pas largement adoptées dans la pratique clinique. D'autres recherches sont nécessaires pour explorer les obstacles à l'adoption de lignes directrices pour la prise en charge des voies aériennes tant par les anesthésiologistes que par les départements d'anesthésie.

Keywords difficult airway · intubation · guideline · anesthesia

Complications of airway management may lead to significant morbidity and mortality. ${ }^{1}$ Between 2007 and 2016, the Canadian Medical Protective Association (CMPA) closed 46 legal actions focused on difficulties in airway management. ${ }^{2}$ Clinical experts were critical of the care provided in $85 \%$ of cases, with most cases leading to serious brain injury or death. Themes identified as contributory to poor outcomes included preparation, situational awareness, team coordination, and communication. These themes are similar to those reported in the most recent American Society of Anesthesiologists (ASA) closed claims analysis and echo concerns highlighted in the 4th National Audit Project (NAP4) from the United Kingdom, the largest study of major morbidity and mortality associated with airway management, now a decade old. ${ }^{3}$ To address some of these areas for improvement, the NAP4 report strongly recommended all anesthetic departments endorse an explicit policy for management of the difficult airway. ${ }^{1}$

Cognitive aids, including algorithms and checklists, have been successfully utilized in healthcare to establish a consistently high standard of baseline performance for both routine and crisis situations. ${ }^{4}$ In a crisis situation, such as cardiac arrest or unanticipated difficult airway, timecritical integration of technical and non-technical skills is required for effective patient care. Guidelines, in conjunction with cognitive aids, emphasize fundamental principles of patient care (i.e., early defibrillation or maintaining oxygenation) while providing a systematic structure for decision-making during a high-stakes, highstress event.

A recent review identified 38 published airway management algorithms. ${ }^{5}$ Among these publications are consensus guidelines for the management of the difficult airway developed by a number of recognized expert airway organizations, including the Canadian Airway Focus Group (CAFG), the Difficult Airway Society (DAS) in the United Kingdom, and the ASA in the United States. ${ }^{6-9}$ With a steadily increasing number of difficult airway algorithms available, it is unclear if these practice recommendations are being endorsed at regional, local or, individual levels.

In this study, we sought to examine local and regional culture of clinical guideline use for management of the unanticipated difficult airway within a hospital partnership in Southwestern Ontario. Our primary outcome measure was the self-reported use of a published clinical practice guideline. Secondary outcomes included the reported effectiveness of dissemination and implementation of existing CAFG clinical practice recommendations, the use of guidelines in clinical education of airway management, and the self-reported confidence in skills required for difficult airway management.

\section{Methods}

The University of Western Ontario's Health Sciences Research Ethics Board approved the study protocol (HSREB 108657, 02/2017). London Health Sciences Centre (LHSC) and St. Joseph's Health Care London (SJHC) form a tertiary academic healthcare partnership affiliated with the Schulich School of Medicine and Dentistry in London, Ontario. This academic centre 
partners with 20 anesthesia departments in Southwestern Ontario to form a healthcare partnership.

Participants

In this study, we targeted two populations: a local population of consultant and trainee anesthesiologists working at LHSC and SJHC, and a regional population of anesthesia department heads within the healthcare partnership. Of note, LHSC is composed of two hospitals with a department head at each site.

We elected to forego a large-scale online survey of all anesthesiologists in Southwestern Ontario because low response rates have negatively impacted result validity in the past. ${ }^{10}$ A focused survey of the local population provided a feasible, in-depth evaluation of current practice within a diverse department. As this survey intended to examine guideline use by anesthesiologists within Southwestern Ontario, trainee anesthesiologists with clinical fellowships were excluded from the survey because of parochial influence on clinical practice. The survey of regional departmental heads sought to establish the magnitude of endorsement of consistent approaches during unanticipated difficult airway management, including coordinated efforts in education and implementation of best evidence and practice.

\section{Survey development}

We generated survey questions through literature review and interviews with potential respondents. To ensure consistent interpretation and appropriateness of content, we conducted pre-testing on the trainee anesthesiologists with clinical fellowships who were excluded from the sample population.

Local and regional surveys comprised 12 and 15 questions, respectively (eAppendix, Electronic Supplementary Material). Both surveys took an estimated five to ten minutes to complete. The local survey was designed to address the following domains: i) participants' use of clinical guidelines, ii) awareness of national guidelines, and iii) self-assessed confidence in fundamental technical skills. Emergency front of neck access (eFONA) to the airway was selected as a rarely performed technical skill that requires practice to limit skill fade. We acknowledge that competence in other technical skills, such as videolaryngoscopy and supraglottic airway use, is essential to minimizing the need for eFONA- and airway management-related morbidity and mortality. Domains within the regional survey included i) departmental endorsement of clinical guidelines, ii) impact of national guidelines on departmental practice, and iii) departmental strategy to maintain best practice (i.e., departmental airway lead clinician, training).

Survey administration

To increase the response rate, investigators selected different survey modes for local and regional populations.

\section{Local survey}

Distribution of paper surveys was feasible on a local scale, avoiding the burden of online survey fatigue. Anonymity was maintained by use of a drop-box collection scheme. Use of routine departmental communication facilitated survey introduction. Between November 2018 and January 2019, paper surveys were distributed to 74 consultant anesthesiologists and 38 trainees employed at LHSC and SJHC. Seven consultant anesthesiologists were on leave or otherwise unavailable to respond to the survey.

\section{Regional}

All department heads were contacted via telephone to introduce the study. In December 2018, an email with an invitation to participate in the survey was distributed using the online survey tool, SurveyMonkey (SurveyMonkey Inc., San Mateo, CA, USA). If no response was received, an automated email reminder with the survey link was sent two weeks following the initial invitation to participate.

Statistical analysis

Survey results were entered into a Microsoft Excel 2018 spreadsheet (Microsoft Cooperation, Redmond, WA, USA) and descriptive statistics were calculated. Results were presented as percentage of respondents. No inferential statistics were used.

\section{Results}

Local survey

A total of 79/112 responses were received (overall response rate, $70 \%$ ), with a response rate of $74 \%$ (28/38) for trainee anesthesiologists and $68 \%(51 / 74)$ for consultant anesthesiologists.

With regards to personal practice in the management of the unanticipated difficult airway, approximately $80 \%$ (64/ 79) of respondents (75\% [38/51] of consultant and $93 \%$ [26/28] of trainee respondents) reported use of a formal published guideline/algorithm. The most common reason for not using a guideline/algorithm was the opinion that 
airway management should not be standardized. Among consultant anesthesiologists endorsing the use of a practice guideline, the most commonly reported practice guideline utilized was the one published by the CAFG (42\%, 16/38; Figure). Of the 64 respondents who used an airway management guideline, 38\% (24/64) were unsure if the guideline they endorsed was appropriate for use in both rapid sequence and elective intubation.

With regards to dissemination, $72 \%$ (57/79) of all respondents were aware of the published CAFG recommendations. The CAFG guidelines were most commonly disseminated via publication in the Canadian Journal of Anesthesia and local presentation (Table). Approximately half of respondents (43/79) said that dissemination of CAFG recommendations was less than satisfactory. Suggestions for improving dissemination included endorsement by a regional or national association, regular local review of recommendations, and cognitive aids displayed in clinical care areas.

Among trainee anesthesiologists, 25\% (7/28) reported being taught multiple guidelines for difficult airway management. Marked variation was reported regarding the use of airway guidelines in trainee airway management education (Figure). Only half of local trainee anesthesiologists reported having received adequate training in the management of difficult airways.

With respect to technical skill performance, 90\% (71/ 79) of all local respondents reported confidence in the use of videolaryngoscopy. Self-reported confidence by trainee anesthesiologists in performing eFONA was $4 \%(1 / 28)$ for needle cricothyroidotomy and 7\% (2/28) for scalpel cricothyroidotomy. Among local consultant anesthesiologists, 24\% (12/51) and 18\% (9/51) reported confidence in performing needle and scalpel cricothyroidotomy, respectively. Of all respondents, $43 \%$ (34/79) reported that they had never practiced nor
TABLE Methods by which respondents reported exposure to the CAFG guidelines

\begin{tabular}{ll}
\hline Method of dissemination & Number of respondents (\%) \\
\hline Read original manuscript in CJA & $26(51 \%)$ \\
Presentation at national meeting & $11(22 \%)$ \\
Presentation at regional meeting & $4(8 \%)$ \\
Presentation in local hospital & $11(22 \%)$ \\
Word of mouth & $5(10 \%)$ \\
Other & $0(0)$ \\
\hline
\end{tabular}

(Total exceeds $100 \%$ as some respondents reported multiple methods of dissemination.)

performed an eFONA, and only 13\% (10/79) reported that they had practiced an eFONA within the last year.

\section{Regional survey}

The regional response rate was $52 \%$ (11/21). Departmental endorsement of a specific clinical guideline was reported by $36 \%$ (4/11) of respondents, with all reporting the use of the ASA guidelines. Ninety percent of the department heads reported no change in departmental practice attributed to the publication of the CAFG recommendations. None of the departments within the region had a designated airway lead.

\section{Discussion}

In this survey, we report heterogeneity in the use of clinical guidelines for the management of the unanticipated difficult airway in local and regional hospitals. Variation existed within departments and between hospitals.
Figure Reported use of difficult airway guidelines by surveyed anesthesiologists

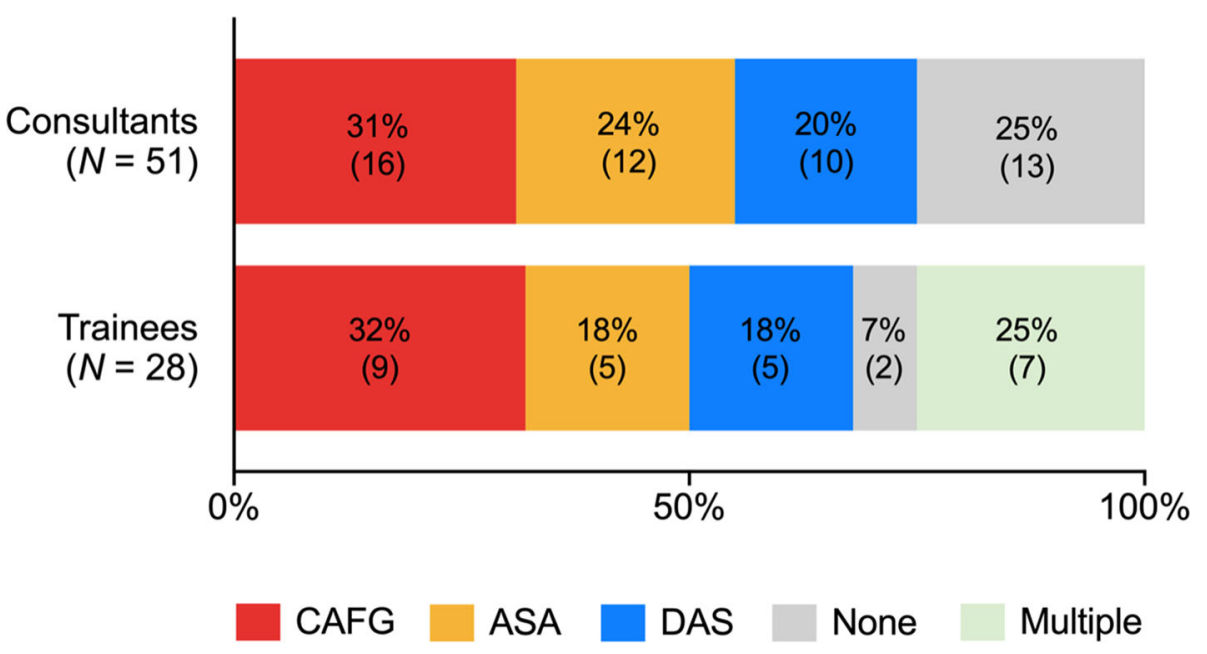


Variation was reported in individual practice as well as teaching of trainee anesthesiologists.

There remains a lack of published evidence relating to the impact of clinical guideline use on airway-related patient outcomes. ${ }^{11}$ The proposed utility of guidelines for unanticipated difficult airway management is largely extrapolated from the long-accepted practice of guideline use in healthcare. As an illustration, use of established advanced life support guidelines and accompanying algorithms is generally recognized as best practice in cardiac arrest management. Evidence shows that improved adherence to advanced life support guidelines is associated with improved patient outcomes. ${ }^{12}$

In this study, the majority of surveyed anesthesiologists reported use of a clinical guideline but with variation in the choice of algorithm employed. With a steady increase in the frequency of published guidelines over recent years, an abundance (or overabundance) of available algorithms may generate confusion among users and negatively impact implementation. ${ }^{5}$

A directed review of published difficult airway management algorithm content and design showed an overwhelmingly similar stepwise approach (i.e., Plan A, B, etc.) when difficulty with airway management is encountered. ${ }^{5}$ Given the similarities in management strategies between published difficult airway guidelines, it may be less important which guideline is adopted on a personal, departmental, or even institutional level. Instead, focusing on departmental or institutional endorsement of a single guideline may improve implementation and adherence.

Approximately two-thirds of local consultant respondents were aware of the CAFG recommendations, while only one-third endorsed use of the CAFG guidelines when encountering difficulty in practice, indicating ineffective implementation strategies. Reasons for this low uptake are likely multifactorial. Approximately $20 \%$ of all respondents reported no use of a formally developed airway guideline for managing the unanticipated difficult airway, citing concern regarding expert consensus for guideline development and oversimplification of clinical care. Emerging themes for improving implementation included guideline endorsement. Notable clinical guidelines, such as those by the ASA and DAS, were developed and presented on behalf of or in conjunction with recognized national anesthesia organizations. The strength of these associations promotes widespread guideline dissemination and encourages a unified approach to complex airway management. Conversely, an absence of formal endorsement on either a provincial or national platform within Canada may contribute to the observed lack of departmental support of a single airway guideline. More research is warranted to further investigate the barriers to airway management guideline implementation in Canada.

Both NAP4 and CMPA examination of closed claims revealed that trainee anesthesiologists were involved in close to one-third of the airway incidents. ${ }^{1,2}$ In our study, it was evident that trainees were taught multiple algorithms, with incomplete mastery of any one algorithm. Over half of trainees were unsure if the airway algorithm they follow is applicable in a rapid sequence intubation, suggesting a lack of in-depth knowledge of algorithm content.

Sound technical skills underpin the foundation of safe airway management. Although there is overlap between technical skills required for routine and difficult airway management, some skills are unique to airway emergencies, i.e., emergency front of neck airway (eFONA). Studies have shown skill decay for rarely performed management techniques. ${ }^{13,14}$ Our results reinforce this issue with an overwhelming self-reported lack of confidence among respondents in performing eFONA. The NAP4 report emphasized the necessity of regular training and rehearsal of airway management skills. 1

The recent COVID-19 pandemic has illustrated the need for coordinated efforts within departments, between hospitals, and across countries to ensure airway preparedness. A network of airway lead clinicians was first launched in the United Kingdom in 2011 following recommendations from the NAP4 report, with strong endorsement from the Royal College of Anaesthetists and the DAS. ${ }^{15}$ Since its inception, this initiative has led to demonstrable advances in airway safety practices across the country. ${ }^{16}$ This pathway for collaboration does not yet exist in Southwestern Ontario.

Limitations of this study are common to many surveys; the small number of respondents made drawing robust conclusions from the results problematic and there was probably inherent responder bias. Yet, the overall response rate was high. The survey was local/regional which limits generalizability. It is unknown if our regional findings represent airway guideline use on a national scale. Further qualitative interviews would have been helpful to explore physician attitudes on guideline adoption and potential barriers to use. Despite these limitations, the present study identified several key areas where focus on guideline endorsement and skills training may translate to improved clinical care in the management of the difficult airway.

With the anticipated release of the newly updated CAFG airway management recommendations, we are developing a regional mastery-based education program to promote guideline implementation. Through sharing of learning opportunities and best practice principles, we hope to establish a regional airway network. This initiative presents many challenges but with the potential for much benefit. 
Author contributions Malcolm Howard contributed to data acquisition, analysis, and interpretation of data and drafting the article. Ruediger Noppens contributed to interpretation of data and drafting the article. Nelson Gonzalez contributed to study design, data acquisition, and drafting the article. Philip M. Jones contributed to study conception and, design and drafting the article. Sonja M. Payne contributed to all aspects of this manuscript, including study conception and design; acquisition, analysis, and interpretation of data, and drafting the article.

Disclosures Dr. R. Noppens and Dr. P. Jones are members of the Canadian Airway Focus Group. Dr. P. Jones is the Deputy Editor-inChief of the Canadian Journal of Anesthesia; he was not involved in the handling of this manuscript. The remaining authors have no conflicts of interest to declare.

Funding statement None.

Editorial responsibility This submission was handled by Dr. Stephan K.W. Schwarz, Editor-in-Chief, Canadian Journal of Anesthesia/Journal canadien d'anesthésie.

\section{References}

1. Cook T, Woodall N, Frerk C; Royal College of Anaesthetists; Difficult Airway Society. 4th National Audit Project of the Royal College of Anaesthetists and he Difficult Airway Society. Major complications of airway management in the United Kingdom. Report and findings March 2011. Available from URL: https:// www.nationalauditprojects.org.uk/downloads/NAP4\%20Full\% 20Report.pdf (accessed February 2021).

2. Crosby ET, Duggan LV, Finestone PJ, Liu R, De Gorter $R$, Calder LA. Anesthesiology airway-related medicolegal cases from the Canadian Medical Protection Association. Can J Anesth 2021; 68: 183-95.

3. Joffe AM, Aziz MF, Posner KL, Duggan LV, Mincer SL, Domino $K B$. Management of difficult tracheal intubation: a closed claims analysis. Anesthesiology 2019; 131: 818-29.

4. Hepner DL, Arriaga AF, Cooper JB, et al. Operating room crisis checklists and emergency manuals. Anesthesiology 2017; 127: 384-92.
5. Edelman DA, Perkins EJ, Brewster DJ. Difficult airway management algorithms: a directed review. Anaesthesia 2019; 74: $1175-85$

6. Law JA, Broemling N, Cooper RM, et al. The difficult airway with recommendations for management - part 1 - difficult tracheal intubation encountered in an unconscious/induced patient. Can J Anesth 2013; 60: 1089-118.

7. Law JA, Broemling N, Cooper RM, et al. The difficult airway with recommendations for management - part 2 - the anticipated difficult airway. Can J Anesth 2013; 60: 1119-38.

8. Frerk C, Mitchell VS, McNarry AF, et al. Difficult Airway Society 2015 guidelines for management of unanticipated difficult intubation in adults. Br J Anaesth 2015; 115: 827-48.

9. Apfelbaum JL, Hayberg CA, Caplan RA, et al. Practice guidelines for management of the difficult airway: an updated report by the American Society of Anesthesiologists Task Force on Management of the Difficult Airway. Anesthesiology 2013; 118: 251-70.

10. Cook DA, Wittich CM, Daniels WL, West CP, Harris AM, Beebe $T J$. Incentive and reminder strategies to improve response rate for internet-based physician surveys: a randomized experiment. J Med Internet Res 2016; DOI: https://doi.org/10.2196/jmir.6318.

11. Crosby ET. An evidence-based approach to airway management: is there a role for clinical practice guidelines? Anesthesia 2011; 66: 112-8.

12. McEvoy MD, Field LC, Moore HE, Smalley JC, Nietert PJ, Scarbrough SH. The effect of adherence to ACLS protocols on survival of event in the setting of in-hospital cardiac arrest. Resuscitation 2014; 85: 82-7.

13. Boet $S$, Borges BC, Naik VN, et al. Complex procedural skills are retained for a minimum of $1 \mathrm{yr}$ after a single high-fidelity simulation training session. Br J Anaesth 2011; 107: 533-9.

14. Stromski C, J Jacoby, Rammohan G, Kasarda D. Short and long term skill retention after resident training in cricothyroidotomy. Ann Emerg Med 2005; DOI: https://doi.org/10.1016/j. annemergmed.2005.06.052.

15. McNarry AF, Cook TM, Baker PA, O'Sullivan EP. The airway lead: opportunities to improve institutional and personal preparedness for airway management. Br J Anaesth 2020; 125: e22-4.

16. Cook TM, Woodall N, Frerk C. A national survey of the impact of NAP4 on airway management practice in United Kingdom hospitals: closing the safety gap in anaesthesia, intensive care and the emergency department. Br J Anaesth 2016; 117: 182-90.

Publisher's Note Springer Nature remains neutral with regard to jurisdictional claims in published maps and institutional affiliations. 